\title{
THE EFFECT OF THICKNESS ON THE OPTICAL PROPERTIES OF ENAMIC HYBRID CERAMIC COMPARED TO VITA FELDSPATHIC ONE: IN VITRO STUDY
}

\author{
Ahmad Saif Soliman*, Rabab Mohammed Ibrahim** and Shereen Adel Ameen**
}

\begin{abstract}
Aim of the study: The aim of the study was to evaluate the effect of thickness on the translucency parameter of Enamic hybrid ceramic compared to feldspathic ceramic (Markll) ceramic.

Materials and Methods: Forty ceramic discs were fabricated using IsoMet 4000 (Buehler, USA) from CAD-CAM blocks of vita Enamic $(\mathrm{n}=20)$ and vita Markll ceramic material $(n=20)$. The samples was made into two different thicknesses $(0.8 \mathrm{~mm}-1.5 \mathrm{~mm})$. One standard shade according to vita 3D master shade guide (VITA Zahnfabrik, Bad Sackingen, Germany), (2M2) was used with all the samples. Finishing and polishing procedures carried out through manufacturer instruction for each ceramic material. Translucency parameter were analyzed and measured using a scanning spectrophotometer (UV- Shimadzu 3101 PC, Chiyoda-ku, Tokyo, Japan). Color measurements $\left(\mathrm{L}^{*}, \mathrm{a}^{*}\right.$ and $\left.\mathrm{b}^{*}\right)$ to the samples placed against an ideal white background (CIE $\mathrm{L}^{*}=96.68, \mathrm{a}^{*}=\_0.18$, and $\left.\mathrm{b}^{*}=\_0.22\right)$ and against an ideal black background $\left(\right.$ CIE $\mathrm{L}^{*}=1.15$, $\mathrm{a}^{*}=\_0.11$, and $\mathrm{b}^{*}=\_0.50$ ) relative to the standard illuminant D65. All the samples was subjected to measurements for each material type, different thicknesses, the same observer carried out the measurements. Three readings was taken and the mean values were tabulated and analyzed. Three way-ANOVA was used to study the effect of ceramic materials and thickness on Translucency Parameter (TP). Tukey's post-hoc test was used for pair-wise comparison between the means when ANOVA test is significant. One-Way ANOVA used to compare between the interaction between variables for mean Translucency parameters.
\end{abstract}

Results: Vita Mark II feldspathic ceramic (18.74 \pm 3.33$)$ showed the highest statistical significant mean translucency value compared to Vita Enamic hybrid ceramic $(12.59 \pm 2.96)$ at $\mathrm{p} \leq 0.001$ * Thickness of $0.8 \mathrm{~mm}(18.31 \pm 3.71)$ showed the highest statistical significant mean translucency value compared to $1.5 \mathrm{~mm}$ thickness $(13.02 \pm 3.34)$ at $\mathrm{p} \leq 0.001 *$.

Conclusions: Vita Mark II Feldspathic ceramic are more translucent than Vita Enamic Hybrid ceramic. Increasing the thickness as well as thermocycling decrease the translucency parameter for both ceramic materials.

* Mcs Fixed Prosthodontics, Faculty of Oral and Dental Medicine, Cairo University

** Professor of Fixed Prosthodontics, Faculty of Oral and Dental Medicine, Cairo University 


\section{INTRODUCTION}

Chair side Computer Aided Design! Manufacturing (CAD\CAM) is recently gaining rapid popularity because its allow the dentist to eliminate the need for a technician to certain laboratory steps, and obtain a constant quality of work. Ceramic material has been advocated rather than metal ceramic restorations because of their excellent esthetic and acceptable mechanical properties; esthetic matching of ceramic restoration with the adjacent teeth should involve shape, texture and optical properties.

The use of a ceramic rather than a metal core permits greater light transmission within a crown, thereby improving the color and translucency of a restoration. Clinically, it is important that ceramic restorations reproduce the translucency and color of the natural teeth. Translucency considered a controlling factor in esthetic of ceramic restoration. The perceived color of natural teeth is a result of light reflected from the enamel surface, in addition to the effect of light scattering within enamel and dentin before it is ultimately reflected back.

Color stability is affected by physical and chemical factor that challenge the color shade over time. Repetitive expansion and contraction stresses induced by temperature changes are claimed to be important physical factor that affect color shade. The translucency in ceramics resulted from different crystalline composition, so it depends on the type of the ceramic material. The translucency is also affected by the thickness of the ceramic material.

The similarity of the translucent parameters of natural tooth enamel, dentine and ceramic restoration could provide a better optical match. The chemical differences in grains and grain boundaries yield a higher level of light absorption and scattering, which may affect final optical outcome of the restoration. The aim of this study was to evaluate the effect of different thicknesses $(0.8 \mathrm{~mm}-1.5 \mathrm{~mm})$ on the optical properties (translucency parameter TP) of
Hybrid Ceramic compared to Feldspathic Porcelain. The research hypothesis of the study was that alteration of ceramic thickness and material could have an impact on their optical properties.

\section{MATERIALS AND METHODS}

Forty ceramic discs were fabricated using IsoMet 4000 (Buehler, USA) from CAD-CAM blocks of vita Enamic $(n=20)$ and vita Markll ceramic material $(n=20)$.

\section{Fabricating the samples}

Each ceramic sample has a diagonal length of $18.4 \mathrm{~mm}$, Perimeter $52 \mathrm{~mm}$, length $12 \mathrm{~mm}$ and width $14 \mathrm{~mm}$. Milling was made into two different thicknesses $(0.8 \mathrm{~mm}-1.5 \mathrm{~mm})$. For standardization, one standard shade according to vita 3D master shade guide (VITA Zahnfabrik, Bad Sackingen, Germany), (2M2) was used with all the samples. The IsoMet microsaw 4000 (Buehler, USA), is a linear precision saw cut materials with minimal specimen deformation and low kerf loss (the material removed during sectioning, roughly equal to the blade width) with a cutting speed $2500 \mathrm{rpm}$, feeding rate $15 \mathrm{~mm} /$ min. Cooling system contains water : anti corrosive agent $(2: 1)$. A diamond-cutting disc of 8 -inch diameter and $0.6 \mathrm{~mm}$ thickness was used.

\section{Finishing and polishing}

After ultrasonic cleaning the feldspathic ceramic (VITABLOCS Mark II) samples were finished using AL2O coated flexible discs (Sof-Lex discs, 3M Espe, USA), in descending order of grit size (black, dark blue, medium blue, light blue) whilst exerting low pressure and ensuring sufficient cooling with water. High-gloss polishing of the ceramic surface was done with Occlubrush (Hawe Neos, Switzerland) and diamond polishing paste (Ultra II polishing paste, Shofu, USA). Polishing was made using low speed range $(15,000 \mathrm{rpm})$ with intermittent pressure (without water-cooling). While hybrid ceramic (Vita Enamic) samples were finished and high 
gloss polished using Vita Enamic Polishing set, (VITA Zahnfabrik, Bad Sackingen, Germany), after ultrasonic cleaning and exerting intermittent slight pressure at low speed rang $(15,000 \mathrm{rpm})$.

\section{Sample size and allocation}

We are planning a study to examine the effect of Thickness on optical properties of Hybrid ceramic compared to Feldspathic one. If we assume that the main effect size of the two materials is 0.5 (medium effect), we will use two different thicknesses in each group and the measurements will be repeated 2 times (after thermo cycling). We will need to study total 40 subjects to be able to reject the hypothesis that the population means of the experimental and control groups are equal with probability (power) 0.85 . The Type I error probability associated with this test of this hypothesis is 0.05 .

A total of forty samples were divided into two equal groups (twenty for each) according to the ceramic material, namely:

- Group One (I): Hybrid ceramic (Vita Enamic).

- Group Two (II): Feldspathic ceramic (VITABLOCS Mark II).

The ceramic groups were ordered randomly into four equal sub groups (ten for each) according to thickness, namely:

- Sub Group A: Feldspathic ceramic (VITABLOCS Mark II) with $0.8 \mathrm{~mm}$ thickness.

- Sub Group B: Hybrid ceramic (Vita Enamic) with $1.5 \mathrm{~mm}$ thickness.

- Sub Group C: Feldspathic ceramic (VITABLOCS Mark II) with $1.5 \mathrm{~mm}$ thickness.

- Sub Group D: Hybrid ceramic (Vita Enamic) with $0.8 \mathrm{~mm}$ thickness.

For randomization, the samples ordered into sub groups randomly, each sub group was numbered from A and ascending, then they were ordered by the web site (http://www.random.org/) into four equal sub groups. Each sub group has 10 samples which has been numbered from 1 to 10 , and sealed with a sealable bag to ensure effective blinding (allocation concealment) during translucency measurement.

\section{Translucency parameter (TP) measurements}

Color and translucency parameter (TP) were analyzed and measured using a bench top scanning spectrophotometer (UV- Shimadzu 3101 PC, Chiyoda-ku, Tokyo, Japan). All the samples was subjected to color measurements for each material type, different thicknesses, the same observer carried out the measurements. Three readings was taken and the mean values were tabulated and analyzed. An integrating sphere of $60 \mathrm{~mm}$ diameter was used and attached in the sample compartment of the spectrophotometer to measure diffuse transmittance and diffuse reflectance of the sample with the use of beam switching software.

The sphere is $\mathrm{Ba} \mathrm{SO}_{4}$ in wall coated. The integrating sphere opening is $18 \mathrm{~mm}$ diameter on reflection side. The sample beam is at incident angle of $0^{\circ}$ and the reference beam at $8^{\circ}$. With the use of beam switching function, the diffuse reflectance can be obtained using the sample-side position and the $8^{\circ}$ specular reflectance is obtained using the reference side position. Color measurements $\left(\mathrm{L}^{*}\right.$, $a^{*}$ and $\left.b^{*}\right)$ to the samples placed against an ideal white background (CIE L* $=96.68, a^{*}=\_0.18$, and $\left.\mathrm{b}^{*}=\_0.22\right)$ and against an ideal black background $\left(\mathrm{CIE} \mathrm{L*}=1.15, \mathrm{a}^{*}=\_0.11\right.$, and $\left.\mathrm{b}^{*}=\_0.50\right)$ relative to the standard illuminant D65 on a reflection spectrophotometer (UV- Shimadzu 3101 PC, Japan). The translucency parameters was calculated via the following equation:

$\mathrm{TP}=\left[\left(\mathrm{LB}^{*}{ }_{-} \mathrm{LW}^{*}\right)^{2}+\left(\mathrm{aB} * \mathrm{aW}^{*}\right)^{2}+\left(\mathrm{bB}^{*}{ }_{-} \mathrm{bW} \mathrm{W}^{2}\right]^{0.5}\right.$

where the subscript $B$ refers to the color parameters against a black background and the subscript $\mathrm{W}$ refers to those against a white background ${ }^{10}$. 


\section{Statistical analysis}

Data presented as means and standard deviation (SD) values. Three way-ANOVA was used to study the effect of ceramic materials and thickness on mean Translucency parameters. Tukey's post-hoc test was used for pair-wise comparison between the means when ANOVA test is significant. One-Way ANOVA used to compare between the interaction between variables for mean Translucency. Statistical analysis was performed with IBM ${ }^{\circledR}$ SPSS $®$ (SPSS Inc., IBM Corporation, NY, USA) Statistics Version 22 for Windows.

\section{RESULTS}

Effect of different ceramic materials on translucency parameter (TP) value: Mean and standard deviation (SD) for the translucency value for different ceramic materials regardless of other variables showed that Vita Mark II feldspathic ceramic (18.74 \pm 3.33$)$ showed the highest statistical significant mean translucency value compared to Vita Enamic (12.59 \pm 2.96$)$ at $\mathrm{p} \leq 0.001 *$.

Effect of different thicknesses on translucency parameter (TP) value: Mean and standard deviation (SD) for the translucency value for different thicknesses regardless of other variables showed that a thickness of $0.8 \mathrm{~mm}(18.31 \pm 3.71)$ showed the highest statistical significant mean translucency value compared to a $1.5 \mathrm{~mm}$ thickness $(13.02 \pm 3.34)$ at $\mathrm{p} \leq 0.001 *$. It is clear that increasing the ceramic material thickness will decrease the translucency values significantly.

\section{DISCUSSION}

Esthetic restoration is the most needed role in dentistry to ensure maximum patient satisfaction and reproduce the natural teeth form, color and shape. Esthetic outcome of the dental restoration included reproducing the color and shape of the natural tooth; translucency has been the controlling factor through the esthetic restoration as its permit passage of light and give life like natural tooth esthetic. Because enamel and dentin have natural translucency, reproduction of the optical properties of natural teeth should be considered when fabricating new restorations ${ }^{1}$. Obtaining a translucency that gives a life like effect to the restoration is essential, in addition to matching the shape and the texture with the adjacent natural teeth ${ }^{2}$.

The science of ceramic material has been increasing concern in esthetic dentistry, new materials fabricated and introduced on the field with a new applications and limitations. Hybrid ceramic is one of the new materials of esthetic restoration, it contains the combination of the advantages of gaining esthetic outcome of the ceramic and the resiliency of the polymer. Nevertheless, the clinical performance of indirect composites is still inferior to the performance of indirect ceramic restorations considering marginal adaptation, color match and anatomic form, so integration may give an opportunity of tacking the advantages of both materials.

The study compared the hybrid ceramic (vita Enamic) to the feldspathic ceramic (Vita Mark II) as they both feldspathic based, but with a polymer network for the hybrid ceramic. Besides, feldspathic ceramic considered as the most esthetic restoration that mimic the natural tooth in term of color, translucency and metamerism².

The variation of thickness has been used to mimic the clinical applications and indications. Thickness of $0.8 \mathrm{~mm}$, which could be used in case of veneer restoration, $1.5 \mathrm{~mm}$ thickness that may be used in case of full coverage crown.

Instrumental color measurement forms the basis of the three-dimensional, CIE color space, which based on the three attributes of color, hue, value and chroma, which embraces the concept of standard observers and standard illuminants. Several color 
spaces have been developed, the CIE L*a*b* color space is the most popular and can be used to describe numerically the color difference $(\Delta \mathrm{E})^{7}$. Spectrophotometers are amongst the most accurate, useful and flexible instruments for overall color matching in dentistry ${ }^{8}$.

Color measurements $\left(\mathrm{L}^{*}, \mathrm{a}^{*}\right.$ and $\left.\mathrm{b}^{*}\right)$ to the samples placed against an ideal white background $\left(\mathrm{CIE} \mathrm{L*}=96.68, \mathrm{a}^{*}=\_0.18\right.$, and $\left.\mathrm{b}^{*}=\_0.22\right)$ and against an ideal black background (CIE L* $=1.15$, $\mathrm{a}^{*}=\_0.11$, and $\left.\mathrm{b}^{*}=\_0.50\right)$ relative to the standard illuminant D65 on a reflection spectrophotometer (UV-Shimadzu 3101 PC, Japan). The translucency parameters was calculated via the following equation:

$\mathrm{TP}=\left[\left(\mathrm{LB}^{*}{ }_{-} \mathrm{LW}^{*}\right) 2+\left(\mathrm{aB}^{*}{ }_{-} \mathrm{aW}^{*}\right) 2+\left(\mathrm{bB}^{*}{ }_{-}\right.\right.$ $\left.\left.\mathrm{bW}^{*}\right) 2\right] 0.5$

where the subscript $\mathrm{B}$ refers to the color parameters against a black background and the subscript $\mathrm{W}$ refers to those against a white background10.

Concerning the translucency parameter (TP) results, Vita Mark II feldspathic ceramic showed a significant higher mean translucency value than Vita Enamic hybrid ceramic. The crystal volume, refractive index and the crystalline content contribute to this difference. This is may be related to the fact that translucency is affected by crystal or filler content within the ceramic composition and particles compactness in the structure ${ }^{10}$. Vita Enamic contains a polymer particle filtrated within feldspathic network, which enable a fewer amount of light transmission through the material ${ }^{11}$, whereas Vita Mark II is only composed of a feldspathic base that are not compacted and contains no filler, thus enables a greater amount of light transmission and higher translucency value. These findings were in agreement with Barizon et al.(2014) ${ }^{(6)}$, Kursoglu et al. $(2015)^{(10)}$.
Whatever the type of the material, a thickness of 0.8 shows higher significant translucency level than 1.5 thickness. This is because increasing the material thickness will induce greater amount of light scattering and eventually decreasing the translucency level ${ }^{10}$. The results showed that both ceramic materials with different composition and different thickness will alter the optical properties, thus the study hypothesis was accepted.

\section{CONCLUSION}

Ceramic material composition and thickness are factors that should be considered during shade matching and fabricating esthetic dental restoration. Vita Mark II Feldspathic ceramic are more translucent than Vita Enamic Hybrid ceramic. Increasing the thickness decrease the translucency parameter for both ceramic materials.

\section{ACKNOWLGMENT}

Special gratitude to Dr. Benjamin A. Just, DDS, CDT. Head of Clinical Affairs Department (VITA Zahnfabrik, Bad Sackingen, Germany).

\section{REFERENCE}

1. Xiong F, Chao Y, Zhu Z. (2008). Translucency of newly extracted maxillary central incisors at nine locations. J Prosthet Dent;100:11-7.

2. Bottino M.A, Faria R and Valandro L.F., Percepcao. (2009). Esteticaem Proteses Livres de Metal em Dentes Naturais e Implantes, (Aesthetic Metal Free Prosthetics in Natural Teeth and Implants) ArtesMedicas, Sao Paulo, Brazil, $1^{\text {st }}$ edition, No. 2, pp.119-287.

3. Abduo, J., Lyons, K., \& Bennamoun, M. (2014). Trends in computer-aided manufacturing in prosthodontics: a review of the available streams. International Journal of Dentistry, 2014,783948 .

4. T. Miyazaki and Y. Hotta (2011). "CAD/CAM systems available for the fabrication of crown and bridge restorations," Australian Dental Journal, Vol.56, No.1, pp.97-106. 
5. Coldea A, Swain MV, Thiel N. (2013). Mechanical properties of polymer-infiltrated-ceramic-network materials. Dent Mater 29:419-426.

6. Barizon, K.T.L., Bergeron, C., Vargas, M. A., Qian, F., Cobb, D.S., Gratton, D.G., \& Geraldeli, S. (2014). Ceramic materials for porcelain veneers: part II. Effect of material, shade, and thickness on translucency. The Journal of Prosthetic Dentistry, 112(4), 864-70. J. Prosdent. 2014.05.016

7. Burkinshaw, S. M. (2004). Colour in relation to dentistry. Fundamentals of colour science. British Dental Journal, 196(1), 33-41.

8. Chu, S.J., Trushkowsky, R.D., \& Paravina, R.D. (2010). Dental color matching instruments and systems. Review of clinical and research aspects. Journal of Dentistry, 38 Suppl 2, e2-16. J Dent. 2010.07.001.

9. Khashayar, G., Bain, P. a, Salari, S., Dozic, A., Kleverlaan, C. J., \&Feilzer, A. J. (2014). Perceptibility and acceptability thresholds for colour differences in dentistry. Journal of Dentistry, 42(6), 637-44. J. Dent. 2013.11.017.

10. Kursoglu, P., Karagoz Motro, P.F., \& Kazazoglu, E. (2015). Translucency of ceramic material in different core-veneer combinations. The Journal of Prosthetic Dentistry, 113(1), 48-53. J. Prosdent. 2014.07.011

11. Della Bona, A., Nogueira, A. D, Pecho, O. E. (2014). Optical properties of CAD-CAM ceramic systems. Journal of Dentistry, 42(9), 1202-9. 